\title{
Phenomenology of meandering of a straight river
}

\author{
Sk Zeeshan Ali ${ }^{*}$ and Subhasish Dey \\ Department of Civil Engineering, Indian Institute of Technology Kharagpur, Kharagpur 721302, \\ India
}

\begin{abstract}
In this study, at first, we analyse the linear stability of a straight river. We find that the natural perturbation modes maintain an equilibrium state by confining themselves to a threshold wavenumber band. The effects of river aspect ratio, Shields number and relative roughness number on the wavenumber band are studied. Then, we present a phenomenological concept to probe the initiation of meandering of a straight river, which is governed by the counter-rotational motion of neighbouring large-scale eddies in succession to create the processes of alternating erosion and deposition of sediment grains of the riverbed. This concept is deemed to have adequately explained by a mathematical framework stemming from the turbulence phenomenology to obtain a quantitative insight. It is revealed that at the initiation of meandering of a river, the longitudinal riverbed slope obeys a universal scaling law with the river width, flow discharge and sediment grain size forming the riverbed. This universal scaling law is validated by the experimental data obtained from the natural and model rivers.
\end{abstract}

\section{Introduction}

Meandering rivers are ubiquitous in earth-like planetary surfaces. Since past, numerous attempts were made to probe the cause of meandering of a river. Several concepts including earth's revolution [1], riverbed instability [2-6], helicoidal flow [7], excess flow energy [8] and macro-turbulent eddies [9] were proposed so far. Despite these concepts, the exact mechanism of the meandering of a straight river remains a vexing phenomenon.

Lane [10] proposed that for nearly straight to meandering rivers, the longitudinal riverbed slope $S$ can be expressed as a function of flow discharge $Q$ in the form of $S=a Q^{b}$, where $a$ and $b$ are empirical constants. Then, Henderson [11] introduced the effects of sediment grain size to refine Lane's [10] empirical formula. However, such relationships exclusively stand on empirical foundation and thus, they are dimensionally inhomogeneous inviting uncertainties. Interestingly, Yalin [9] introduced the concept of macro-turbulent eddies to explain the meandering of a river. He argued that the longitudinal length scale of macro-turbulent eddies is approximately equal to the longitudinal length of alternate bars in a straight river. He considered the length scale of the macro-turbulent eddies as six times the river width. Although, this concept provided a qualitative idea of the meandering of a

\footnotetext{
* Corresponding author: skzeeshanali@,iitkgp.ac.in
} 
river; a precise physical mechanism and a quantification of this phenomenon is still unknown. In fact, the initiation of meandering was found to be fundamentally dependent on the formation of steady or slowly migrating alternate bars in a river [12-14]. Despite several mechanisms of the river meandering dynamics reported in earlier studies [15-18], a precise phenomenological framework of the initiation of meandering is still unknown.

In subsequent sections, a linear stability analysis of a straight river is first performed. Then, the phenomenology of the initiation of meandering of a straight river is described. Finally, concluding remarks are made.

\section{Linear stability analysis}

\subsection{Mathematical analysis}

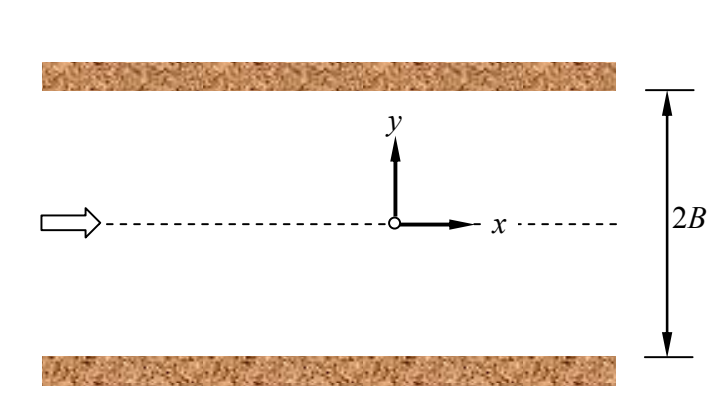

$$
\text { Wers }
$$
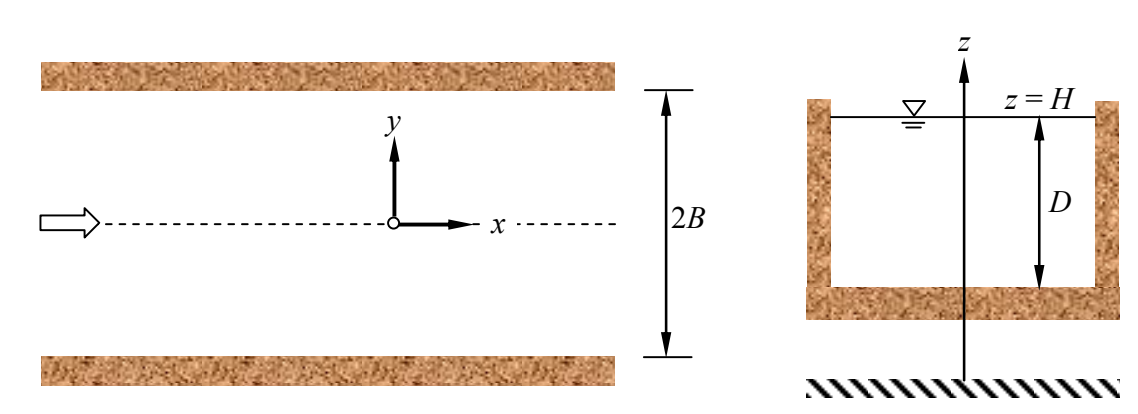

Fig. 1. Sketch of a straight river and the coordinate system.

Figure 1 depicts the schematic of a straight river with a constant width of $2 B$, flowing over an erodible granular bed, confined to two parallel riverbanks. With reference to the Cartesian coordinate system $(x, y)$, where $x$ is the streamwise distance and $y$ is the lateral distance from the river centreline, the depth-averaged velocity components in $(x, y)$ are $(U$, $V$ ), respectively. Further, $D$ and $H$ denote the flow depth and the free surface elevation from a known reference level, respectively, and $z$ represents the vertical coordinate from the reference level. The bed shear stress components and the volumetric sediment flux components in $(x, y)$ are denoted by $\left(T_{x}, T_{y}\right)$ and $\left(Q_{x}, Q_{y}\right)$, respectively.

The flow continuity equation is [19]

$$
\frac{\partial}{\partial \hat{x}}(\hat{D} \hat{U})+\frac{\partial}{\partial \hat{y}}(\hat{D} \hat{V})=0
$$

The flow momentum equations are [19]

$$
\begin{aligned}
& \hat{U} \frac{\partial \hat{U}}{\partial \hat{x}}+\hat{V} \frac{\partial \hat{U}}{\partial \hat{y}}+\frac{\partial \hat{H}}{\partial \hat{x}}+A \frac{\hat{T}_{x}}{\hat{D}}=0 \\
& \hat{U} \frac{\partial \hat{V}}{\partial \hat{x}}+\hat{V} \frac{\partial \hat{V}}{\partial \hat{y}}+\frac{\partial \hat{H}}{\partial \hat{y}}+A \frac{\hat{T}_{y}}{\hat{D}}=0
\end{aligned}
$$

The sediment flux continuity equation is [20] 


$$
\frac{\partial}{\partial \hat{t}}\left(F^{2} \hat{H}-\hat{D}\right)+\frac{\partial \Phi_{x}}{\partial \hat{x}}+\frac{\partial \Phi_{y}}{\partial \hat{y}}=0
$$

where $(\hat{x}, \hat{y})=(x, y) / B, \hat{D}=D / D_{m},(\hat{U}, \hat{V})=(U, V) / U_{m}, \hat{H}=H^{2} /\left(F^{2} D_{m}\right), F[=$ $\left.U_{m} /\left(g D_{m}\right)^{1 / 2}\right]$ is the flow Froude number in the unperturbed state, $g$ is the gravitational acceleration, $A\left[=B / D_{m}\right]$ is the river aspect ratio, $\left(\hat{T}_{x}, \hat{T}_{y}\right)=\left(T_{x}, T_{y}\right) /\left(\rho_{f} U_{m}^{2}\right), \rho_{f}$ is the mass density of fluid, $\hat{t}=t U_{m} Q_{r} / B, t$ is the time, $Q_{r}=\left(\Delta g d^{3}\right)^{1 / 2} /\left[\left(1-\rho_{0}\right) U_{m} D_{m}\right], \Delta\left[=\left(\rho_{g}-\rho_{f}\right) / \rho_{f}\right]$ is the submerged relative density of sediment grains, $\rho_{g}$ is the mass density of sediment grains, $d$ is the median size of sediment grains, $\rho_{0}$ is the porosity of sediment, $\left(\Phi_{x}, \Phi_{y}\right)=$ $\left(Q_{x}, Q_{y}\right) /\left(\Delta g d^{3}\right)^{1 / 2}$ and subscript " $m$ " refers to the quantities linked with the unperturbed uniform flow.

The bed shear stress components $\left(\hat{T}_{x}, \hat{T}_{y}\right)$ are given as $\left(\hat{T}_{x}, \hat{T}_{y}\right)=(f / 8)(\hat{U}, \hat{V})\left(\hat{U}^{2}+\right.$ $\left.\hat{V}^{2}\right)^{1 / 2}$, where $f$ is the Darcy-Weisbach friction factor. The $f$ is expressed as [21]

$$
\frac{1}{f^{1 / 2}}\left\lfloor=\frac{1}{8^{1 / 2}} \frac{U}{u_{*}}\right\rfloor=-0.86 \ln \left\lfloor\left(\frac{\hat{k}_{s}}{14.8 \hat{D}}\right)^{1.1}+\frac{2.51}{R f^{1 / 2}}\right\rfloor
$$

where $u_{*}$ is the shear velocity, $\hat{k}_{s}=k_{s} / D_{m}, k_{s}$ is the bed roughness height, $R(=4 U D / v)$ is the Reynolds number and $v$ is the coefficient of kinematic viscosity of fluid. The $k_{s}$ is considered as $k_{s}=2.5 d$ [22].

The $\left(\Phi_{x}, \Phi_{y}\right)$ are given as $\left(\Phi_{x}, \Phi_{y}\right)=\Phi(\cos \beta, \sin \beta)$, where $\beta$ is the angle created by the grain trajectory with the streamwise direction. The $\beta$ is expressed as $\beta=\sin ^{-1}$ \{sin $\delta-$ $\left.\left[r /(A \Theta)^{1 / 2}\right](\partial / \partial \hat{y})\left(F^{2} \hat{H}-\hat{D}\right)\right\}[23]$, where $\delta$ is the angle between the shear stress vector and the streamwise direction, $\Theta$ is the Shields number and $r$ is a coefficient $(=0.5)$ [24]. The $\delta$ is given as $\delta=\sin ^{-1}\left[\hat{V} /\left(\hat{U}^{2}+\hat{V}^{2}\right)^{1 / 2}\right]$. Considering the bedload transport as the prevailing mode of sediment transport, the $\Phi$ is expressed as $\Phi=\left(\Theta-\Theta_{c}\right)^{1.5}[25]$, where $\Theta_{c}$ is the threshold Shields number. The $\Theta_{c}$ is determined from the empirical formulae proposed by Cao et al. [26].

To perform the stability analysis, the primitive variables are expressed as $(\hat{U}, \hat{V}, \hat{H}$, $\hat{D})=\left(1,0, \hat{H}_{m}, 1\right)+\varepsilon[(\tilde{U}, \tilde{V}, \tilde{H}, \tilde{D}) \exp \mathrm{i}(\hat{k} \hat{x}-\omega \hat{t})][6]$, where $\varepsilon$ is $O(1), \mathrm{i}=(-1)^{1 / 2}$, $\hat{k}$ is the nondimensional wavenumber and $-\mathrm{i} \omega$ is the complex quantity. Therefore, the expansions of the $\hat{T}_{x}$ and the $\Phi$ produce $\hat{T}_{x}=(f / 8)\left\{1+\varepsilon\left[\left(\tilde{U} c_{1}+\tilde{D} c_{2}\right) \exp \mathrm{i}(\hat{k} \hat{x}-\omega \hat{t})\right]\right\}$ and $\Phi=\Phi_{m}\left\{1+\varepsilon\left[\left(\tilde{U} c_{3}+\tilde{D} c_{4}\right) \exp \mathrm{i}(\hat{k} \hat{x}-\omega \hat{t})\right]\right\}$, where $c_{1}=2\left[1-\left(\Theta_{m} / f_{m}\right)(\partial f / \partial \Theta)\right]^{-1}, c_{2}=$ $\left(1 / f_{m}\right)(\partial f / \partial \hat{D})\left[1-\left(\Theta_{m} / f_{m}\right)(\partial f / \partial \Theta)\right]^{-1}, c_{3}=\left(\Theta_{m} / \Phi_{m}\right)(\partial \Phi / \partial \Theta) c_{1}$ and $c_{4}=\left(\Theta_{m} / \Phi_{m}\right)(\partial \Phi / \partial \Theta) c_{2}+$ $\left(1 / \Phi_{m}\right)(\partial \Phi / \partial \hat{D})$. Considering these aspects, the governing equations [Eqs. (1)-(4)] at $O(\varepsilon)$ can be expanded and after some algebra, we find:

$$
\frac{-\mathrm{i} \omega\left\{F^{2}\left[\left(a_{11}-a_{14}\right) a_{22} a_{31}\right]-\left[a_{13} a_{22} a_{31}-a_{11} M^{2}\right]\right\}}{\Phi_{m}}
$$




$$
=\left(a_{13} a_{22} a_{31}-a_{11} M^{2}\right)\left[a_{44} M^{2}-a_{45}+\frac{a_{14} a_{41}}{a_{11}}\right]+\left(a_{11}-a_{14}\right) a_{22} a_{31}\left[\left(a_{43}-\frac{1}{a_{22}}\right) M^{2}+\frac{a_{13} a_{41}}{a_{11}}\right]
$$

where $a_{11}=\Psi c_{1}+\mathrm{i} \hat{k}, \Psi=A f_{m} / 8, a_{13}=a_{31}=a_{34}=\mathrm{i} \hat{k}, a_{14}=\Psi\left(c_{2}-1\right), a_{22}=\Psi+\mathrm{i} \hat{k}, a_{41}=$ $\mathrm{i} \hat{k} c_{3}$, and $a_{43}=-r F^{2} /(A \Theta)^{1 / 2}$.

\subsection{Results and discussion}

To show the graphical illustration, the mass densities of fluid and grains are taken as $\rho_{f}=$ $1000 \mathrm{~kg} \mathrm{~m}^{-3}$ and $\rho_{g}=2650 \mathrm{~kg} \mathrm{~m}^{-3}$, respectively. In addition, the shear Reynolds number $R *$ $=500$ is considered to characterize the hydraulically rough flow regime. Note that the condition $\operatorname{Re}(-\mathrm{i} \omega)>0$ indicates an exponential growth rate, while the condition $\operatorname{Re}(-\mathrm{i} \omega)<0$ describes an exponential decay rate. Figure $2(a)-2(c)$ presents the $\operatorname{Re}(-\mathrm{i} \omega)$ and $\operatorname{Im}(-\mathrm{i} \omega)$ as a function of nondimensional wavenumber $\hat{k}$ computed from Eq. (6) for different values of river aspect ratio $A$, Shields number $\Theta$ and relative roughness number $\zeta$. In Fig. 2(a), the evolutions of $\operatorname{Re}(-\mathrm{i} \omega)$ and $\operatorname{Im}(-\mathrm{i} \omega)$ are studied for different aspect ratios $A$ by keeping the relative roughness number $\zeta(=0.005)$ and the Shields number $\Theta(=0.2)$ as a constant. For a given $\hat{k}$ with $\hat{k}<0.1$, the $\operatorname{Re}(-\mathrm{i} \omega)$ has a decay with a decrease in $A$. By contrast, for a given $\hat{k}$ with $\hat{k}>0.1$, the $\operatorname{Re}(-\mathrm{i} \omega)$ grows with $A$. The variations of $\operatorname{Im}(-\mathrm{i} \omega)$ with $\hat{k}$ suggest that for a given $\hat{k}$ with $\hat{k} \ll 0.1$, the frequency of excitation is almost independent of $A$. On the other hand, for a given $\hat{k}$ with $\hat{k}>0.1$, the frequency of excitation quickly decays with a decrease in $A$. In Fig. 2(b), the evolutions of $\operatorname{Re}(-\mathrm{i} \omega)$ and $\operatorname{Im}(-\mathrm{i} \omega)$ are examined for different Shields numbers $\Theta$ by keeping the aspect ratio $A(=30)$ and the relative roughness number $\zeta(=0.005)$ as a constant. For a specific $\Theta$, the straight river responds rapidly to the external perturbations, because the variations of $\operatorname{Re}(-\mathrm{i} \omega)$ with $\hat{k}$ reveal an exponential growth or decay, excluding in the vicinity of $\hat{k} \approx 0.1$. For a given $\hat{k}$ with $\hat{k} \ll 0.1$, the $\operatorname{Re}(-\mathrm{i} \omega)$ decays with $\Theta$. On the contrary, for a given $\hat{k}$ with $\hat{k}>0.1$, the $\operatorname{Re}(-\mathrm{i} \omega)$ increases with $\Theta$. However, when $\hat{k}$ approaches unity, the $\operatorname{Re}(-\mathrm{i} \omega)$, for a larger $\Theta$, rapidly grows with a high frequency. The variations of $\operatorname{Im}(-\mathrm{i} \omega)$ with $\hat{k}$ show that for a given $\hat{k}$ with $\hat{k} \ll 0.1$, the frequency of excitation is practically invariant of $\Theta$. However, for a given $\hat{k}$ with $\hat{k}>0.1$, the frequency of excitation increases with $\Theta$. In Fig. 2(c), the evolutions of $\operatorname{Re}(-\mathrm{i} \omega)$ and $\operatorname{Im}(-\mathrm{i} \omega)$ are presented for different relative roughness numbers $\zeta$ by keeping the aspect ratio $A(=30)$ and the Shields number $\Theta(=0.2)$ as a constant. For a given $\hat{k}$ with $\hat{k}<0.1$, the $\operatorname{Re}(-\mathrm{i} \omega)$ diminishes with $\zeta$. The variations of $\operatorname{Im}(-\mathrm{i} \omega)$ with $\hat{k}$ indicate that for a given $\hat{k}$ with $\hat{k} \ll 0.1$, the frequency of excitation is independent of $\zeta$. Furthermore, for a given $\hat{k}$ with $0.1<\hat{k}<0.5$, the frequency of excitation slowly reduces with a decrease $\zeta$. On the contrary, for a given $\hat{k}$ with $0.5<\hat{k}<1$, the frequency of excitation increases with $\zeta$.

Interestingly, in Fig. 2(a)-2(c), for a small window of wavenumbers $\hat{k}$, there exists a wavenumber band $\hat{k}$, for which both $\operatorname{Re}(-\mathrm{i} \omega)$ and $\operatorname{Im}(-\mathrm{i} \omega)$ are quite small, let us say -0.05 $<[\operatorname{Re}(-\mathrm{i} \omega), \operatorname{Im}(-\mathrm{i} \omega)]<0.05$. Such wavenumber band can be thought as a threshold 
wavenumber band for which the natural perturbation modes are at equilibrium state (that is, they neither grow nor decay). In fact, a straight river with an unperturbed bed becomes unstable when large scale bed perturbations grow for a wide range of external variables. In this situation, there exists a specific range of wavenumbers that try to preserve the straight course of the river resulting in the initiation of meandering.

(a)

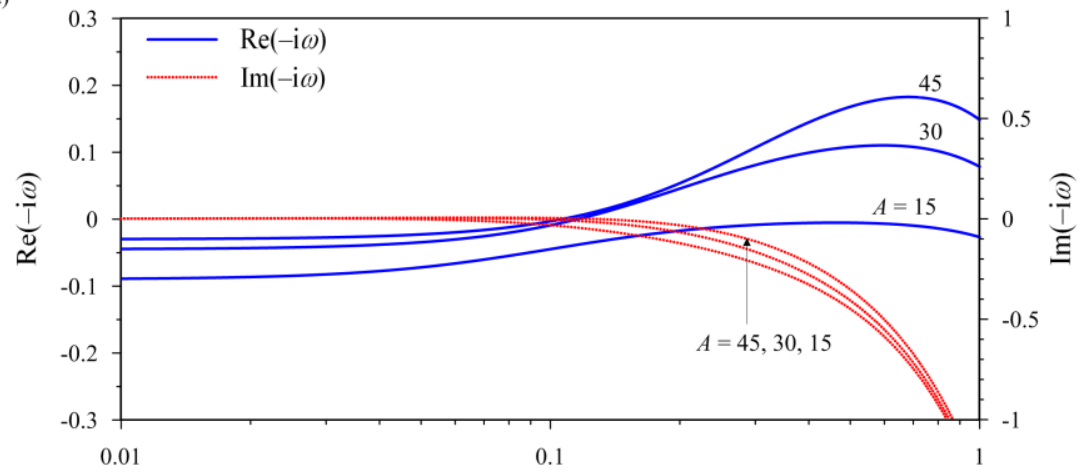

(b)

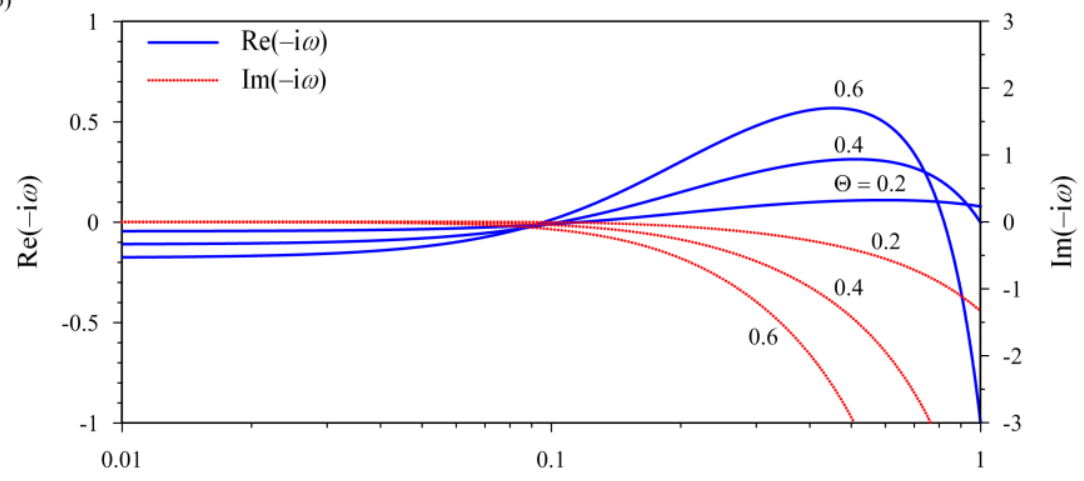

(c)

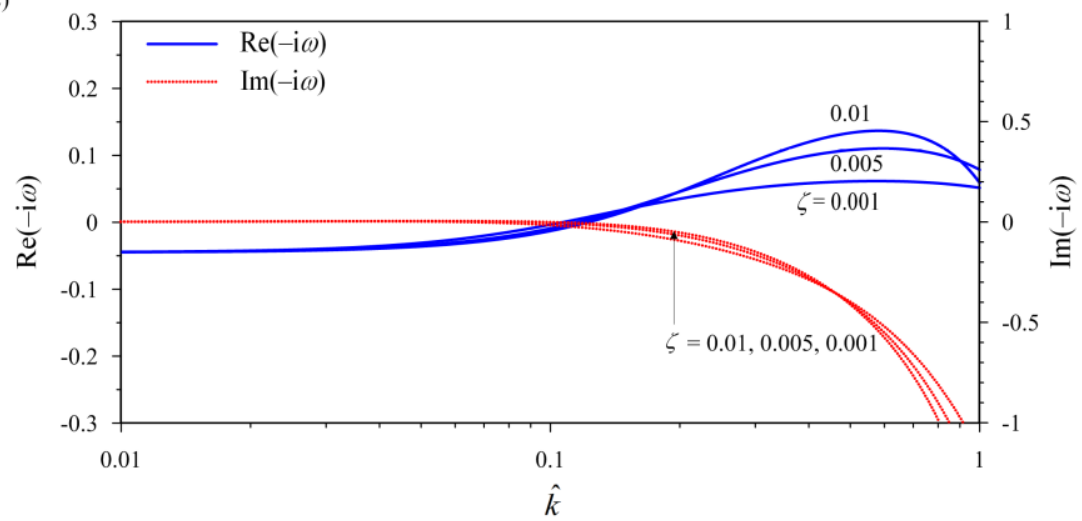

Fig. 2. The $\operatorname{Re}(-\mathrm{i} \omega)$ and $\operatorname{Im}(-\mathrm{i} \omega)$ as a function of nondimensional wavenumber $\hat{k}$ for different values of (a) aspect ratio $A=15,30$ and 45 (b) Shields number $\Theta=0.2,0.4$ and 0.6 and (c) relative roughness number $\zeta=0.001,0.005$ and 0.01 .

The stability analysis clearly shows the response of the river to the external perturbations by revealing the presence of threshold wavenumber band. However, to gain a 
physical insight into the initiation of meandering, a conceptual framework of this phenomenon is presented in the next section. This framework is further explained by a mathematical model stemming from the turbulence phenomenology.

\section{Conceptual framework}

\subsection{Theory}

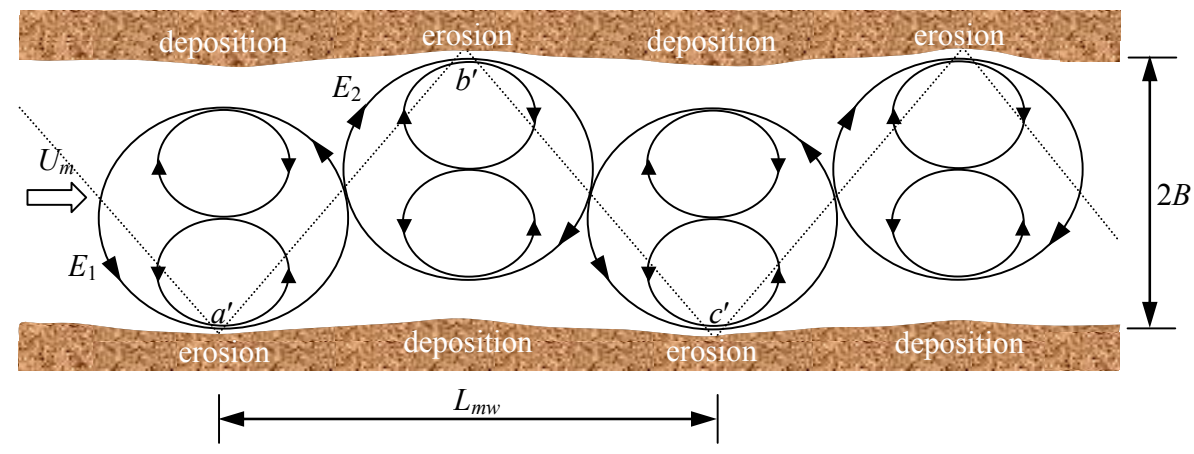

Fig. 3. Conceptual representation of the initiation of meandering of a straight river.

Figure 3 shows the conceptual mechanism of the initiation of meandering. Let us consider a straight river having a width $2 B$, an unperturbed flow depth $D_{m}$, a streamwise bed slope $S$, an averaged flow velocity $U_{m}$ and a flow discharge $Q$. The riverbed consists of sediment grains of median size $d$. The near-bed turbulent structure is principally anisotropic in nature, suggesting that the velocity fluctuations have directional preference. This turbulence anisotropy leads to the development of turbulence induced secondary currents, known as the secondary currents of Prandtl's second kind [25]. Figure 3 shows that the large-scale turbulent eddy $E_{1}$ tends to move randomly towards one of the riverbanks (in this case, it moves towards the right bank). This highly intermittent lateral shifting of the large-scale turbulent eddies was experimentally observed in a rough turbulent flow [27]. Since this eddy rotates counter-clockwise, it erodes the sediment grains near the right bank. The eroded grains are then deposited at the opposite riverbank. The neighbouring large-scale turbulent eddy $E_{2}$, triggered by the motion of the eddy $E_{1}$, therefore has a clockwise rotation with a slight lateral shift towards the left bank. As a consequence, the sediment grains eroded from the left bank are deposited at the opposite side. In this fashion, the processes of alternate erosion and deposition take place. This phenomenon of the motion of eddies is analogous to that of solid spheres, arranged in a row in succession, confined to two parallel boundaries. When the first sphere is given a counter-clockwise rotation with a slight shift towards the right boundary, subsequently the next sphere displays a clockwise rotation with an equal shift towards the left boundary. For the remaining spheres, a similar alternate process of rotation and shift takes place. The processes of alternate erosion and deposition of grains in the riverbed by the large-scale eddies can be therefore understood from this physical mechanism. The advent of such successive eddies creates gravity waves with a speed $U_{w}$ proportional to $\left(g D_{m}\right)^{1 / 2}$ [28]. The waves successively traverse the riverbank (dotted lines in figure 3), making a meandering wavelength $L_{m w}$.

To obtain a quantitative criterion of the meandering of a straight river, the concept of equal periodicity is applied [28]. This concept states that the time required for the gravity 
wave to traverse the distance between the alternate bars is the same as that for the mean flow to cover a straight path between the alternate bars. Fundamentally, the mean flow velocity has a close link with the turbulent energy spectrum. Applying the turbulence phenomenology, the mean velocity can be expressed as $U_{m}=a_{1}\left(g D_{m} S\right)^{1 / 2}\left(d / D_{m}\right)^{-1 / 6}[29]$, where $a_{1}$ is a coefficient. The time taken by the flow to traverse a straight path between the alternate bars is $L_{m w} / U_{m}$. On the other hand, the time taken by the wave to traverse this distance is $\left(L_{m w}^{2}+16 B^{2}\right)^{1 / 2} / U_{w}$. The $U_{w}$ is expressed as $U_{w}=a_{2}\left(g D_{m}\right)^{1 / 2}$ [28], where $a_{2}$ is a coefficient. The flow discharge is given as $Q=2 B D_{m} U_{m}=2 a_{1} B D_{m}\left(g D_{m} S\right)^{1 / 2}\left(d / D_{m}\right)^{-1 / 6}$. Therefore, equating the above time periods, results in:

$$
S \propto B^{2 / 9} Q^{-2 / 9} d^{1 / 3} g^{1 / 9}
$$

\subsection{Results and discussion}

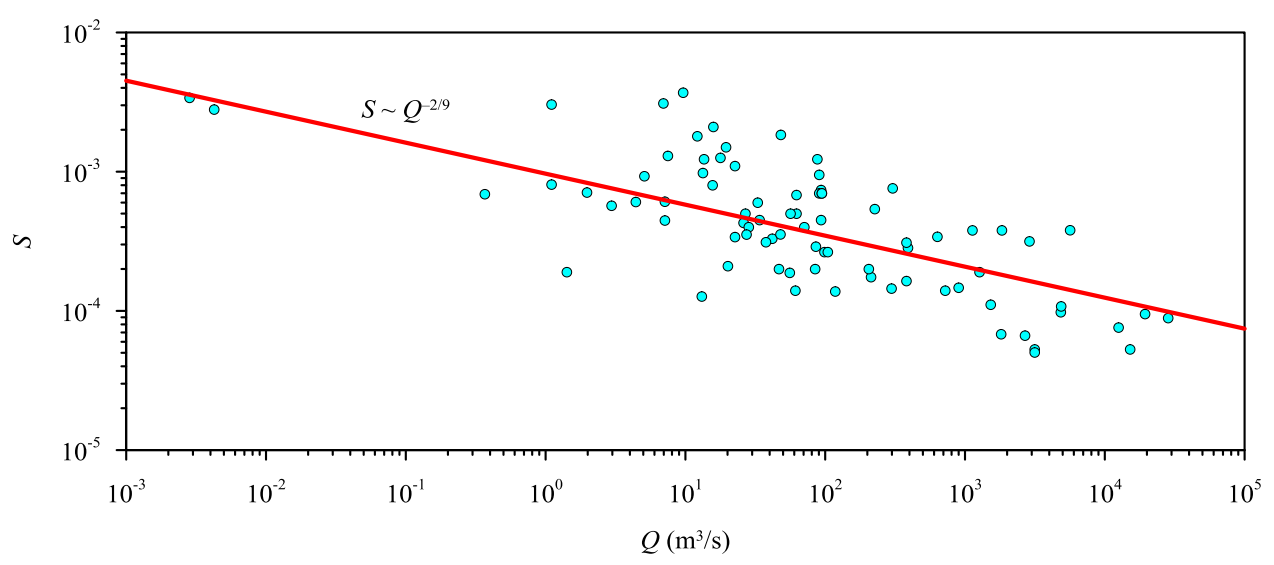

Fig. 4. Variation of streamwise riverbed slope $S$ with flow discharge $Q$.

Equation (7) provides a scaling law of the initiation of meandering of a straight river. It is apparent that for constant values of the gravitational acceleration, river width and sediment grain size, the threshold slope is proportional to the " $-2 / 9$ "-th power of the flow discharge. Figure 6 displays the functional representation of $S(Q)$ and the experimental data collected from several natural and model rivers $[10,30]$, featured by approximately straight to highly meanders. The mean slope of the plotted data band that obeys a " $-2 / 9$ " scaling law is shown by the straight line. Note that the overall scatter of the experimental data is owing to the variability of river widths and grain sizes. Previously, Lane [10] proposed the following formula: $S=7 \times 10^{-4} Q^{-0.25}$. Therefore, the " $-2 / 9$ " (= -0.22$)$ scaling law derived from this study satisfactorily corresponds to that proposed by Lane [10].

\section{Conclusion}

A linear stability analysis of a straight river shows that there exists a threshold wavenumber band for which the natural perturbation modes maintain an equilibrium state over a broad range of river aspect ratios, Shields numbers and relative roughness numbers. The initiation of meandering of a river is governed by the turbulent flow with a counter-rotational motion of the neighbouring large-scale eddies in succession to generate the processes of alternate erosion and deposition of sediment grains. This concept is explained by a mathematical model stemming from the perspective of the turbulence phenomenology to provide a 
quantitative insight into the meandering of a straight river. It is found that the longitudinal riverbed slope, river width, flow discharge and median grain size are connected by a unique scaling law which has a satisfactory agreement with the experimental data.

\section{References}

1. A. Einstein, Die Naturwissenschaften 14, 1-3 (1926)

2. J. F. Friedkin, United States Waterways Experiment Station, Vicksburg (1945)

3. R. A. Callander, J. Fluid Mech. 36, 465-480 (1969)

4. F. Engelund, O. Skovgaard, J. Fluid Mech. 57, 289-302 (1973)

5. J. Fredsøe, J. Fluid Mech. 84, 609-624 (1978)

6. P. Blondeaux, G. Seminara, J. Fluid Mech. 157, 449-470 (1985)

7. W. F. Tanner, J. Geophys. Res. 65, 993-995 (1960)

8. C. T. Yang, J. Hydrol. 13, 231-253 (1971)

9. M. S. Yalin, Mechanics of sediment transport (Pergamon, 1977)

10. E. W. Lane, United States Army Engineer Division, Missouri River Division, Corps of Engineers, Omaha, Nebraska (1957)

11. F. M. Henderson, Trans. Am. Soc. Civ. Eng. 128, 657-686 (1963)

12. K. W. Olesen, Communications on hydraulics, 1983-01, Delft University of Technology (1983)

13. K. W. Olesen, PhD thesis, Delft University of Technology (1987)

14. G. Duró, A. Crosato, P. Tassi, Adv. Water Resour. 93, 21-38 (2016)

15. G. Zolezzi, G. Seminara, J. Fluid Mech. 438, 183-211 (2001)

16. A. Crosato, E. Mosselman, Water Resour. Res. 45, W03424 (2009)

17. K. Asahi, Y. Shimizu, J. Nelson, G. Parker, J. Geophy. Res. 118, 2208-2229 (2013)

18. G. Seminara, M. Tubino, Water Resources Monograph, AGU, eds. S. Ikeda and G. Parker (2013)

19. B. de Saint-Venant, Comptes Rendus de l'Academic des Sciences, vol 73, Paris, pp 147-154, 237-240 (1871)

20. F. M. Exner, Sitzungsberichte der Akademie der Wissenschaften 134, 165-203 (1925)

21. C. F. Colebrook, C. M. White, Proc. R. Soc. Lond. A 161, 367-381 (1937)

22. F. Engelund, E. Hansen, Acta Polytech. Scand. 35, 1-100 (1966)

23. S. Ikeda, J. Hydraul. Div. 108, 1097-1099 (1982)

24. A. M. Talmon, N. Struiksma, M. C. L. M. van Mierlo, J. Hydraul. Res. 33, 495-517 (1995)

25. S. Dey, Fluvial hydrodynamics: hydrodynamic and sediment transport phenomena (Springer, 2014)

26. Z. Cao, G. Pender, J. Meng, J. Hydraul. Eng. 132, 1097-1099 (2006)

27. I. Albayrak, U. Lemmin, J. Hydraul. Eng. 137, 1318-1334 (2011)

28. P. W. Werner, Trans. Am. Geophys. Union 32, 898-902 (1951)

29. G. Gioia, F. A. Bombardelli, Phys. Rev. Lett. 88, 014501 (2002)

30. L. B. Leopold, M. G. Wolman, Professional paper 282-B, United States Geological Survey, Washington, DC (1957) 